\title{
Feature Level Fusion in Multimodal Biometric Authentication System
}

\author{
M.Fathima Nadheen \\ PG Scholar, Department of IT, \\ SSN College of Engineering \\ Chennai, India.
}

\author{
S.Poornima \\ Assistant Professor, Department of IT, \\ SSN College of Engineering, \\ Chennai, India.
}

\begin{abstract}
Multimodal systems integrate multiple sources of human information to ensure high level security. Multimodal biometric systems increase the recognition rate of the biometric systems either by reducing the false acceptance rate (FAR) or false rejection rate (FRR). Multiple biometric traits can be combined at feature level. Feature level fusion increases the reliability of the system by preventing the biometric template from modification. In the proposed system, feature level fusion is employed to fuse the feature vectors of iris and ear extracted by Principal Component Analysis technique, which also reduces the dimension of the feature vectors. Finally matching is performed by comparing the test fused feature vectors with all training images using distance measure. This system is developed to study and analyze, whether the performance of multimodal biometric system is improved over unimodal biometric system by attaining $93 \%$ success rate when fusion is inclined.
\end{abstract}

\section{Keywords}

Feature level fusion, Genuine Acceptance Rate, Morphological operation, Principal Component Analysis.

\section{INTRODUCTION}

Biometric systems prevent the security breaches by protecting the unauthenticated person from accessing the secure information. Traditional biometric systems are unimodal i.e. they rely on single biometrics for authentication. Unimodal biometric system developed using finger print is subjected to spoofing attack, in which the thumb impression can be easily forged. Similarly in face recognition system intra-class variability problem occurs due to aging, variation in facial expression. These problems can be resolved in multimodal biometric systems.

Multimodal biometric system employs various fusion rules for fusing the biometric modalities. Fusion [1] of biometric modalities takes place at various stages in the biometric recognition system. Some fusion techniques commonly employed in biometric authentication system are as follows:

1. Sensor level fusion or Image level fusion:

This type of fusion is carried out immediately after capturing the modalities from sensor. In this case, multiple modalities obtained from different sensors will be combined together and treated as a single biometric modality.

2. Feature level fusion:

In this fusion, the features are extracted from the all biometrics traits. Later the extracted features can be combined together into a final feature vector of higher dimension.

3. Score level fusion:
This level of fusion uses less information for fusion, in which the similarity scores of the individual biometrics are generated and the scores are fused together for recognition.

\section{Decision level fusion:}

The decisions obtained from the individual authentication system are fused to authenticate a person.

The first two levels of fusion (Sensor, feature) methods take place prior to matching stage, whereas score and decision level fusion plays their role at the later stage of matching.

The work in this paper describes about the feature level fusion, while developing a bimodal recognition system using a new combination of biometric traits, Iris and Ear. Feature vectors from the two traits are obtained by applying Principal component Analysis technique. Fusion at earlier stage provides more information for identifying a person. Hence, Feature level fusion is applied over the feature vectors to obtain a final fused feature vector. The paper is organized as follows.

\section{Section 2 - Related Works}

Section 3 - Implementation details of the proposed system

Section 4 - Results and Discussion

Section 5 - Conclusion

\section{RELATED WORKS}

Several multimodal biometric systems were developed by combining different biometric modalities to provide human security and good identification. Fusion plays a major role in multimodal authentication. Score level fusion [2-3] is the simplest form of fusion technique that uses the similarity scores of the modalities for fusion. Different normalization scheme were employed to normalize the scores to improve the performance of the system. Nazmeen Bibi Boodoo, R K Subramanian [4] extracted the features of ear and face by PCA method, then fused the modalities at decision level by AND rule, increased the accuracy of authentication system, as well as reduced the FAR to $0 \%$. But fusion at decision and score level use only less information from the biometric for authentication. Richa Mishra, V. Pathak [5] concatenated the features of iris and ear images (Haar wavelet coefficients) obtained from Haar wavelet transform into a fused feature vector of higher dimension. In most scenarios, Feature level fusion becomes hard to implement if the features considered are incompatible with each other. Various Normalization schemes [6-7] were proposed to make feature vector compatible prior to fuse the features. The dimensionality of fused feature vectors is reduced by feature reduction 
techniques [8]. Feature level fusion using correlation filter [9] is employed to fuse the features obtained from incompatible biometric domain, in which PSR value is used to distinguish the recognized/non-authenticated person.

Ear recognition provides more structural information for identifying a person. The study of human ear [10] reveals that ear also has standard parts like face. Unlike face, ear does not change with emotions and the color is uniformly distributed. Apart from manual cropping of ear images, there were some works which automated the ear region detection. Choras [11] detected the contour of the ear region by finding the difference between maximum value and minimum value of pixel intensity and then compared it against the threshold, followed by Geometric feature extraction that considers only a few geometric features. In [12], improved Adaboost algorithm was employed for ear detection followed by feature extraction from ear image using Neighborhood Preserving Embedding (NPE) algorithm. It is observed that some part of ear region poses higher amount of information, especially the inner ear region.

In Reference [13], iris region is segmented by morphological operation and it also reduced the computation time by avoiding the normalization of iris region. PCA is employed over DWT coefficients to extract the feature vectors. In order to reduce the computation time for feature extraction, Haar wavelet [14] is used, in which the iris region is localized by canny edge detection and circular Hough transform. A. Basit et al [15] proposed an iris recognition method in which pupil boundary is detected first followed by iris boundary detection. (ii) Eigen vectors are calculated over the normalized iris image to represent the feature vectors.

\section{PROPOSED DESIGN}

The bimodal recognition system implemented at Feature level fusion involves the following processing steps.

- $\quad$ Preprocessing of Ear and Iris

- Feature extraction from Ear and Iris

- Feature level Fusion of Ear and Iris feature vectors

- Verification

The overall block diagram of the feature level biometric system is shown in Figure 1, which explains the regular biometric processing stages preprocessing, segmentation, normalization, feature extraction and matching. Additionally, the other concentrated area is fusion after feature extraction.

\subsection{Unimodal Biometric}

Two unique unimodal biometric, Iris and Ear were chosen for this work. These two traits were used individually for recognition in different applications. Iris and ear region has unique patterns that are invariant to aging. Both of the traits prevent the user from having direct contact with sensing device. Ear and Iris image are surrounded by occluding regions, which must be removed in the preprocessing stage.

\subsection{Preprocessing of Iris and Ear}

The image of the human ear captured by the sensor device contains noise due to its large background. Raw images are difficult to recognize, hence the ear image is preprocessed for easier detection of the ear region from the surrounding area.
Series of morphological operations [14-15] are applied over the raw image of the ear to obtain the edge information. The resultant image is free from noise.

In most of the conventional ear recognition systems, the ear image is cropped manually. The present paper proposes an automated approach for ear detection. Here, the region of interest is extracted by detecting the edges from the raw ear image. Noises in the image makes the edge detection difficult, hence the noise effect was removed by convolving the image with Gaussian operator. Morphological operations analyses the shape of the image by choosing an appropriate structuring element. Dilation residue edge detector determines the edges of the image.

As iris region is the inner part of the eye, it cannot be directly captured using sensor. Hence the eye image must be preprocessed to segment the iris part. Two steps involved in extracting the iris region are (i) Segmentation (ii) Normalization.

\section{Segmentation:}

Iris is segmented by detecting edge map of iris and pupil using canny edge detector. From the estimated edge map, Circular Hough Transform detects the centre and radius of iris and pupil boundary .Eyelid and eyelashes adds noise to the iris, Eyelid region is removed by excluding the region surrounding the iris boundary and the eyelashes are eliminated by threshold.

Normalization:

Its purpose is to maintain same iris texture information regardless of pupil dilation. Normalization approach produces a 2D array using the pixel coordinates within the iris region. Daugman's rubber sheet model [16] performs the mapping of each pixel in the iris into rectangular region.

\subsection{PCA based Feature Extraction}

Feature extraction captures the detailed information about the images which form the basis of recognition system and reduces the image dimension by representing them in the form of feature vectors. Principal Component Analysis [13] is a feature extraction technique that extracts global features from the images. This is an oldest method which computes Eigen values and generates Eigen vectors for extracting the features of the corresponding input. The steps involved in PCA are as follows:

a) Represent all the 2D images in the database as a $1 \mathrm{D}$ row or column vector in the form of matrix.

b) Average across each dimension is calculated that gives the mean.

c) Mean vector is subtracted from each dimension to give a zero mean dataset.

d) Compute the Eigen vector and Eigen values.

e) Choose the first few Eigen vectors corresponding to maximum Eigen values.

f) Eigen vectors represent a pattern in an image which forms the principal component.

g) The final feature vector is obtained by multiplying the Eigen vector with mean subtracted image. 


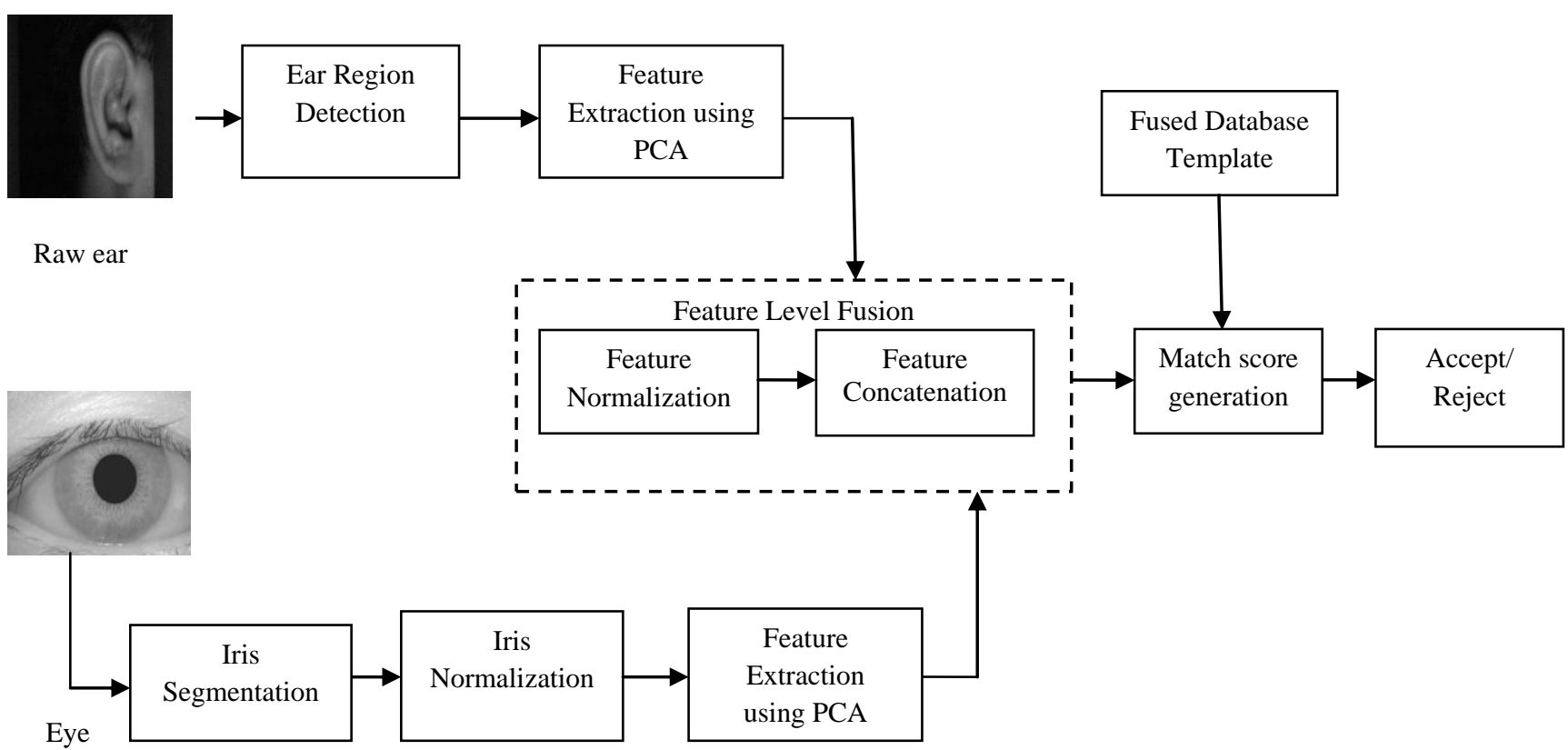

Figure 1. Block diagram of Feature level fusion System

\subsection{Feature Level Fusion}

Feature level fusion has richer information about biometric template. Feature level fusion combines the biometric information prior to matching. This level of fusion also reduces the response time than score level fusion. Feature level fusion is not widely adapted because it is difficult to fuse incompatible feature vectors from multiple modalities. The simplest form of feature level fusion is concatenating the extracted features. Concatenation of feature set may increases the dimensionality of the fused feature vector. Here, the problem of curse of dimensionality is reduced by PCA.

\subsubsection{Normalization of Feature Vector}

The features vectors extracted independently from iris and ear image are incompatible in nature due to variation in their range and distribution. One way to overcome this problem is to normalize the feature vectors by normalization scheme (min-max, z-score, median).Here, min-max normalization scheme is applied, which normalize the feature vectors in the range $[0,1]$. Let $X=\left\{x_{1}, x 2, x 3 \ldots x_{n}\right\}$ be the feature vector, the normalized feature vector $X$ can be represented using minmax normalization (1).

$$
\mathrm{X}^{\prime}=\frac{x i-\min (X)}{\max (X)-\min (X)}
$$

\subsubsection{Fusing the Feature Vector}

The final fused vector is obtained by simple concatenation of normalized feature vectors of iris and ear region into a single fused vector. Let $\mathrm{E}_{\mathrm{i}}=\left\{\mathrm{e}_{1}, \mathrm{e}_{2}, \mathrm{e}_{3} \ldots \mathrm{e}_{\mathrm{n}}\right\}$ and $\mathrm{I}_{\mathrm{i}}=\left\{\mathrm{i}_{1}, \mathrm{i}_{2}, \mathrm{i}_{3} \ldots \mathrm{i}_{\mathrm{n}}\right\}$ be the normalized feature vectors of Ear and iris region. The fused vector (2) is represented as

$$
\text { Fused }_{\text {vector }}=\left[e_{1}, e_{2}, e_{3} \ldots e_{n} i_{1}, i_{2}, i_{3} \ldots i_{n}\right]
$$

\subsection{Verification}

In the testing phase, the ear and eye image of a person is obtained, which are then preprocessed to extract their feature vectors. The feature vectors are fused to form final test feature vector. The test feature vector is compared with final fused vectors of images in the database using any distance measure or similarity measure. Euclidean distance generates the scores by computing the distance between trained and test feature vectors. Smaller distance gives the better match.

\section{RESULTS AND DISCUSSION}

Ear images are collected from IIT Delhi Ear Database (Version 1.0) and iris images from CASIA Version 1.0. The proposed system is tested with 60 subject's multiple samples (approximately 3 per each). When the developed multimodal system is experimented with chosen samples at various level of threshold, the performance obtained is shown in Figure 2 plotted against Genuine Acceptance Rate (GAR) vs. False Acceptance Rate (FAR).

Table 1 FAR, GAR rates of Iris, Ear and Feature level Recognition system

\begin{tabular}{|c|c|c|c|c|c|}
\hline Iris & $\begin{array}{c}\text { Iris } \\
\text { FAR }\end{array}$ & $\begin{array}{c}\text { Ear } \\
\text { FAR }\end{array}$ & $\begin{array}{c}\text { Ear } \\
\text { GAR }\end{array}$ & $\begin{array}{c}\text { Feature } \\
\text { level } \\
\text { fusion } \\
\text { FAR }\end{array}$ & $\begin{array}{c}\text { Feature } \\
\text { level } \\
\text { fusion } \\
\text { GAR }\end{array}$ \\
\hline 0.15 & 87.5 & 0.2 & 90 & 0 & 85 \\
\hline 0.2 & 90 & 0.35 & 92.5 & 0.05 & 90 \\
\hline 0.25 & 92.5 & 0.5 & 95 & 0.1 & 92.5 \\
\hline 0.35 & 95 & 0.65 & 100 & 0.15 & 95 \\
\hline 0.5 & 97.5 & 0.9 & 100 & 0.3 & 97.5 \\
\hline 0.7 & 97.5 & 0.95 & 100 & 0.45 & 100 \\
\hline
\end{tabular}




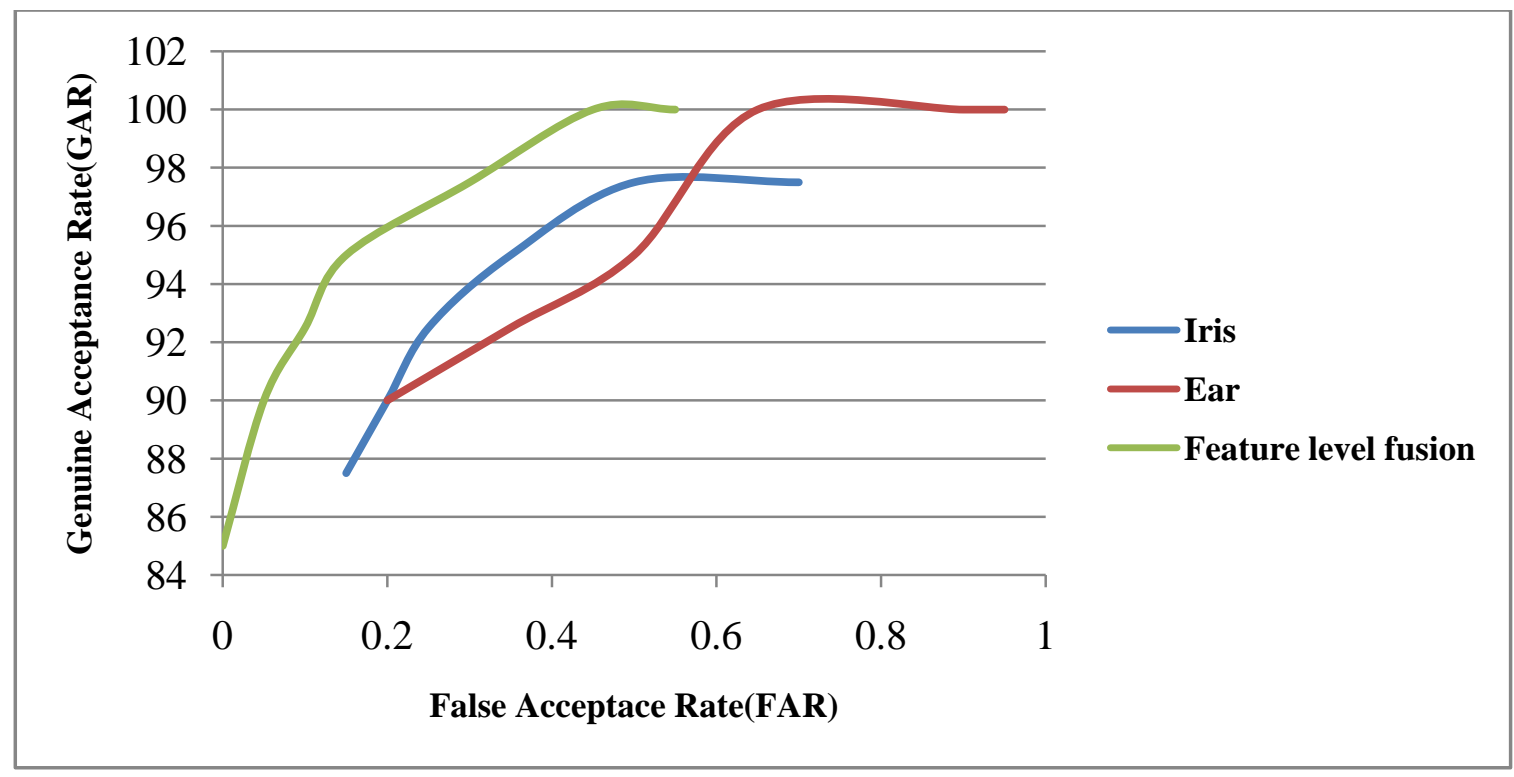

Figure 2 Performance Graph

The green line in the graph indicates that feature level fusion in multimodal system gives higher GAR with minimum FAR than unimodal biometric ear and iris recognition systems. It can be observed from the graph that, for the considered FAR range of 0 to 0.55 , GAR evaluated after fusion is increasingly higher than unimodal iris and ear biometric system. In each case of above performance shown in graph, the GAR becomes stable after certain FAR irrespective of threshold. Table 1 gives the experimental results of FAR and GAR of iris, ear recognition system and feature level multimodal system for the plotted graph in figure 1.

The performance of the system is evaluated using common performance metrics, FAR, FRR (False Rejection Rate). By varying the threshold, the system gives different FAR and FRR.The threshold must be fixed for the system in such a way that it must obtain the minimum Equal Error Rate (EER). Table 2 shows the performance results of our feature level fused system in comparison with unimodal systems of ear and iris. The results show that fusion of these two biometric traits increases the recognition rate rather a single biometric trait used in any authentication system.

Table 2 Performance Evaluation

\begin{tabular}{|c|c|c|c|}
\hline Metric & Iris & Ear & $\begin{array}{c}\text { Feature } \\
\text { Level Fusion }\end{array}$ \\
\hline FAR & 0.15 & 0.2 & 0.05 \\
\hline FRR & 0.12 & 0.1 & 0.075 \\
\hline $\begin{array}{c}\text { Accuracy } \\
(\%)\end{array}$ & 86 & 86 & 93 \\
\hline
\end{tabular}

\section{CONCLUSION}

This paper was discussed about the work done on feature level fusion in multimodal system, which proved itself as a better fusion method for increasing the reliability of the biometric system required for a sensitive authentication and verification for a known or unknown individual. This work might still get improved in its performance rate with another advanced suitable feature extraction method.

\section{REFERENCES}

[1] Ashish Mishra, "Multimodal Biometrics it is: Need for Future Systems," International Journal of Computer Applications, vol. 3, pp. 28-33, June 2010.

[2] Mingxing He, Shi-JinnHorng, Pingzhi Fan, Ray-Shine Run , Rong-Jian Chen , Jui-Lin Lai , Muhammad Khurram Khan, Kevin Octavius Sentosa , "Performance evaluation of score level fusion in multimodal biometric systems," Pattern Recognition, vol. 43, pp. 1789-1800, May 2010.

[3] Shi-Jinn Horng, "An Improved Score Level Fusion in Multimodal Biometric Systems," International Conference on Parallel and Distributed Computing, Applications and Technologies, pp. 239 - 246, Dec 8-11, 2009.

[4] Nazmeen Bibi Boodoo, R K Subramanian, "Robust Multi-biometric Recognition Using Face and Ear Images," International Journal of Computer Science and Information Security, Vol. 6, No. 2, 2009.

[5] R. Mishra, V. Pathak, "Human Recognition using fusion of Iris and Ear Data," International Conference on Methods and Models in Computer Science, pp. 1-5, December 2009.

[6] A. Ross and R. Govindarajan, "Feature Level Fusion in Biometric Systems," SPICE Conference on Biometric Technology for human identification.. II, Vol. 5779, Orlando USA, March 2005, pp. 196-204. 
[7] A. Ross and R. Govindarajan, "Feature Level Fusion Using Hand and Face Biometrics", Proceedings of SPIE Conference on Biometric Technology for Human Identification II, Orlando, USA, pp. 196-204, March 2005.

[8] A. Rattani, D. R. Kisku, M. Bicego, and M. Tistarelli, "Feature Level Fusion of Face and Fingerprint Biometrics," 1st IEEE International Conference on Biometrics, Theory, Applications and Systems, pp. $1-6$, September 2007

[9] Suryanti Awang, Rubiyah Yusof, "Fusion of Face and Signature at the Feature Level by using Correlation Pattern Recognition", World Academy of Science, Engineering and Technology, 2011.

[10] A. Iannarelli. "Ear Identification Forensic Identification Series", Paramount Publishing Company, Fremont, California. 1989.

[11] Michał Choras, "Ear Biometrics Based on Geometrical Feature Extraction," Electronic Letters on Computer Vision and Image Analysis 5, pp. 84-95, 2005.
[12] Li Yuan, Zhi chun Mu, "Ear recognition based on local information fusion," Pattern Recognition Letters 33(2), pp. 182-190, 2012.

[13] Shashi Kumar D R, K B Raja, R. K Chhootaray, Sabyasachi Pattnaik, "PCA based Iris Recognition using DWT,” Int. J. Comp. Tech. Appl., vol 2 (4), pp. 884-893, July-August 2011.

[14] Naveen Singh, Dilip Gandhi, Krishna Pal Singh, "Iris recognition system using a canny edge detection and a circular Hough transform," International Journal of Advances in Engineering \& Technology, May 2011.

[15] A. Basit, M. Y. Javed, M. A. Anjum, "Efficient Iris Recognition Method for Human Identification," proceedings of world academy of science, engineering and technology, vol. 4 feb.2005.

[16] John Daugman, "How Iris Recognition Works", in Proceedings of International Conference on Image Processing, vol.1, pp. I-33- I-36, 2002. 